\title{
Más allá de una teoría general del Estado Capitalista: "el pensamiento fuerte" de América Latina y los desafios del capitalismo global
}

\author{
Beyond a general theory of Capitalist State: the "strong" Latin \\ American thought and the challenges of global capitalism
}

\section{Juan Camilo Arias}

\section{Resumen}

El presente artículo identifica los aportes teóricos que el "pensamiento fuerte" latinoamericano realizó en aras de comprender el Estado capitalista, así como los elementos de dicha tradición que pueden contribuir al entendimiento de las transformaciones estatales que ha implicado la globalización neoliberal. Para ello, se recurre a aspectos de la teoría de la dependencia que abordaron las especificidades del Estado y el capitalismo en la región, así como a los aportes realizados por pensadores como Kaplan y Zavaleta a propósito de los debates planteados por Nikos Poulantzas sobre el Estado capitalista. Por último, se resaltan críticas realizadas a esta corriente de pensamiento por parte de estudiosos del Estado y el capitalismo global, destacando que si bien es necesario replantear la rigidez de categorías como el Norte y el Sur, su legado sigue representando un aporte vigoroso para entender las transformaciones del Estado capitalista en América Latina durante la globalización.

\section{Palabras clave}

Estado Capitalista; Teoría de la Dependencia; Globalización Neoliberal; América Latina.

\begin{abstract}
This paper identifies some theoretical contributions made by the "strong" Latin American thought in order to comprehend the capitalist state, as well as those elements that can contribute to the understanding of state transformations during the neoliberal globalization. To this end, we turn to aspects from Dependency Theory which addressed the specificities of the state and capitalism in the region, as well as the contributions made by thinkers such as Kaplan and Zavaleta regarding the debates prompted by Nikos Poulantzas on the capitalist state. Finally, criticisms made to this current of thought by scholars of the state and Global Capitalism are highlighted, considering that although it is necessary to rethink the rigidity of categories such as the North and the South, the legacy of "strong" Latin American thought continues to represent a vigorous contribution to understanding the transformations of the capitalist state in Latin America during globalization.
\end{abstract}

\section{Keywords}

Capitalist State; Dependency Theory; Neoliberal Globalization; Latin America. 


\section{Introducción}

El presente artículo se propone identificar algunos de los aportes que el "pensamiento fuerte" latinoamericano ha realizado para la comprensión del Estado capitalista en la región, así como aquellos elementos de dicha tradición que puedan contribuir al entendimiento de las transformaciones estatales que ha implicado la globalización neoliberal. En tanto campo de estudios alternativo al institucionalismo y al realismo, cabe señalar que el Estado capitalista se consolidó a partir de las críticas desarrolladas por Nikos Poulantzas (2007, 1991a, 1991b, 1979, 1976, 1973) a la teoría general del Estado y el instrumentalismo marxista a finales de los años 1960s. Asimismo, hizo parte de su intento por completar el vacío de una teoría materialista del Estado en la obra de Marx (1973), lo que le permitió alentar nuevos debates acerca de la especificidad de la forma política del capitalismo y las eventuales transformaciones que presentaría a finales del siglo XX (JESSOP, 2011 y 2007; BARROW, 2000; PANITCH, 1999; SOLO, 1978).

En este decurso, se prestará especial atención a algunas de las contribuciones realizadas desde América Latina con el fin de avanzar en la construcción de una teoría materialista del Estado acorde con las especificidades de la región. Entre otros, perspectivas como las de Kaplan (1983) o Zavaleta (1990), serán consideradas valiosas no solo por haber entrado en discusión con la obra de Poulantzas o el debate alemán de la derivación, sino por haber puesto en el centro de su discusión las realidades políticas específicas de América Latina. Asimismo, se abordará el modo en que algunos estudiosos de la teoría materialista del Estado y el capitalismo global han recibido algunas premisas centrales de la teoría dependentista, con el fin de observar cuáles de sus categorías contribuyen al análisis de la globalización, y cuáles pueden ser reactualizadas a la luz de las complejas transformaciones que han tenido lugar a partir de las tres últimas décadas del siglo XX.

\section{El capitalismo dependiente $y$ el pensamiento fuerte de América Latina}

Si bien la mayor parte de los debates sobre el Estado capitalista han tenido lugar en países industrializados y, por tanto, hay vacíos importantes respecto al modo en que este ha sido teorizado para las formaciones sociales dependientes (JESSOP, 2015), en América Latina se han dado esfuerzos importantes con el propósito de avanzar en la materia e ir más allá del eurocentrismo y las categorías universales. Sin embargo, son varios los desafíos que ha debido enfrentar la elaboración de una teoría 
materialista del Estado acorde con las especificidades de la región, siendo el primero de ellos la ausencia de dicha teoría en el trabajo de Marx. Aunque las alusiones de este pensador fueron profusas respecto a la forma mercancía, la teoría del valor o las contradicciones inherentes al sistema capitalista, las referencias al Estado quedaron más bien dispersas en su obra, pese a la intención manifiesta de destinar uno de los volúmenes del Capital (MARX, 1973) a este asunto (HEINRICH, 2004; BARROW, 2000).

Asimismo, aunque en América Latina la forma política presenta como uno de sus rasgos fundamentales la escisión respecto a la sociedad civil característica del poder político en las sociedades capitalistas (HIRSCH y KANNANKULAM, 2011; GERSTENBERGER, 2011; JESSOP, 2015), esta no se cristalizó históricamente de la misma manera que en las formaciones sociales dominantes (ARICÓ, 2009; KAPLAN, 1983; ZAVALETA, 1990). Entre las razones de esto último, destacan los diferentes modos de inserción al mercado mundial propiciados por un desarrollo desigual del capitalismo, lo cual debe ser tenido en cuenta con el fin de evitar definiciones universales o teleológicas, que resultan imprecisas a la hora de comprender las realidades económicas y políticas de América Latina (MARINI, 2008). De allí que una teoría general del Estado capitalista, igualmente válida para todas las latitudes, condujera al desconocimiento de los rasgos propios que tanto este como el capitalismo han adoptado alrededor del mundo (ZAVALETA, 1990).

Debido a esto último, parte de los esfuerzos de la teoría social latinoamericana se centraron en la identificación y posterior articulación teórica de las especificidades políticas, económicas y culturales de la región. En este sentido, destaca la así denominada teoría de la dependencia que, especialmente entre las décadas de 1960s y 1970s, inspiró a la CEPAL con el fin de comprender las particularidades del desarrollo capitalista en América Latina y las formas sociales que lo componen, más allá de los atributos generales identificados por la teoría marxista o weberiana (ARICÓ, 2009; ZAVALETA, 1990; MARINI, 2008; TORRES-RIVAS, 2008; LECHNER, 1977; FALETO, 1989). Aunque dicha corriente del pensamiento fuerte en la región, como fue denominada con posterioridad debido al compromiso de algunos de sus exponentes con la transformación socialista (CORTÉS, 2013), contó con una diversidad importante de autores y perspectivas, ha destacado como uno de sus tópicos centrales la idea de que las formas dependientes de articulación al mercado internacional, determinaron rasgos estructurales en la economía política de América Latina a partir de la experiencia colonial, los cuales no se diluyeron a pesar de las victorias obtenidas durante las luchas por la Independencia: 
La constitución, durante la primera mitad del siglo XIX, de Estados nacionales formalmente independientes de las metrópolis colonialistas, no redundó en una simétrica autonomía en la definición de los procesos productivos internos. Por el contrario, las articulaciones sociales consecuentes estuvieron marcadas por la continuidad en la inserción subordinada a los centros de poder de los países centrales (THWAITES y OUVIÑA, 2012, p. 65).

Para llegar a esta premisa, que al día de hoy representa uno de los rasgos fundamentales de esta corriente de pensamiento, fue relevante el trabajo de Marini (2008) al afirmar que el tipo de capitalismo instaurado en la región no estaría signado por una suerte de destino precapitalista que, luego de consumar una serie de etapas, permitiría equiparar a los países periféricos con las metrópolis europeas. Por el contrario, se trataría de un tipo específico de capitalismo, basado en la articulación subordinada al mercado mundial que, para sostenerse, requeriría de la internalización de las condiciones de dependencia. Esta última, consistiría entonces en la exportación de bienes primarios obtenidos a través de formas intensivas de explotación del trabajo, que serían posteriormente insertados en cadenas de valor tecnificadas en las metrópolis para su distribución en los mercados internacionales. La dialéctica de la dependencia o, en otras palabras, el ciclo dependiente del capital, no solo determinó las estructuras económicas y políticas de América Latina a partir del siglo XIX, sino que además reprodujo formas desiguales de articulación al mercado mundial. El resultado en el tiempo de este ciclo dependiente del capital, no sería propiamente el desarrollo, sino la profundización de las condiciones de subordinación a través de un tipo sui generis de capitalismo:

Lo que habría que decir es que, aun cuando se trate realmente de un desarrollo insuficiente de las relaciones capitalistas, esa noción [precapitalismo] se refiere a aspectos de una realidad que nunca podrá desarrollarse, por su estructura global y su funcionamiento, de la misma forma como se han desarrollado las economías capitalistas llamadas avanzadas. Por lo tanto lo que se tiene, más que un precapitalismo, es un capitalismo sui generis que sólo cobra sentido si lo contemplamos en la perspectiva del sistema en su conjunto, tanto a nivel nacional como, y principalmente, a nivel internacional (MARINI, 2008, p. 108).

Para la corriente del pensamiento dependendista en América Latina, el hecho de que el capitalismo no fuera visto como un fenómeno que se presentó de la 
misma manera en todas las latitudes, implicaba que la forma Estado asociada con sus distintas expresiones tendría a su vez particularidades insoslayables. Arguyendo un descubrimiento tardío del continente por parte de la teoría materialista, el cual explicaría parcialmente la no identificación de los rasgos específicos de sus estructuras políticas y económicas, Aricó (2009) descartó que dicho vacío fuera resultado del eurocentrismo de Marx. Más importante para el caso, serían las discrepancias teóricas con Hegel (2004 y 2001) a la hora de reconocer la capacidad que tiene el Estado de producir a la sociedad civil (CORTÉS, 2013; MEDINA, 2016) ${ }^{1}$. Este punto de tensión, sin embargo, resultó relevante para la construcción de un concepto del Estado acorde con la región. El caso particular del Estado en Latinoamérica, de acuerdo con Aricó (2009), radicaba en que este no fue el resultado de la condensación de las fuerzas de la sociedad civil y la organización de los mercados internos - como en algunos casos de Europa -, sino la respuesta a impulsos provenientes del exterior. El Estado capitalista en América Latina, fue implantado desde afuera y desempeñó un papel predominantemente productivo en la conformación de las clases sociales y los mercados internos.

En consecuencia, lo que podría considerarse como otro tópico fundamental en la construcción de una teoría materialista del Estado para América Latina - a parte de la condición dependiente del capitalismo en la región-, es justamente el carácter productivo desempeñado por dicha forma política en la articulación y reproducción de la sociedad civil y el mercado en sociedades profundamente heterogéneas (LECHNER, 1977 y 1980; ZAVALETA, 1990; ARICÓ, 2009; FALETO, 1989; KAPLAN, 1983; TORRES-RIVAS, 2008). El Estado no sería para el caso la condensación de la sociedad civil y las fuerzas productivas, sino una condición previa para su existencia. El reconocimiento de este aspecto representó un avance importante respecto a los debates latinoamericanos que tuvieron lugar entre 1950 y 1960, los cuales estuvieron dominados por la influencia althousseriana y la corriente del capitalismo monopolista de Estado, que difuminó el problema de lo político bajo un estructuralismo dogmático que lo limitaba a mero reflejo de la base

\footnotetext{
${ }^{1}$ Este presupuesto respecto a Marx, no obstante, ha sido revaluado a la luz de trabajos recientes como el de Pradella (2015), el cual se remite a manuscritos de los años 1840s para precisar el papel atribuido allí al Estado en el desarrollo capitalista - aspecto que ya había sido abordado tanto por los economistas clásicos como por los mercantilistas -, afirmando que la visión de Marx consistió más bien en una crítica a la comprensión del Estado y el capital como realidades autónomas, sin negar por ejemplo el papel crucial de las relaciones antagónicas entre Estados en la creación del mercado internacional posterior a la Conquista del Nuevo Mundo.
} 
económica. Por el contrario, el reconocimiento del carácter productivo del Estado en América Latina, hizo más complejas las maneras de entender la relación entre las formas económicas y políticas en la región, como lo demuestran en particular los aportes de Kaplan (1983) y Zavaleta (1990).

\section{E1 "Leviatán criollo" o el Estado neocapitalista tardio y dependiente}

Debates como el sostenido a través de la New Left Review entre Miliband y Poulantzas (TARCUS, 1991) o la crítica materialista de este último al concepto del poder en la obra de Foucault (1980), propiciaron importantes reflexiones sobre la especificidad del Estado en América Latina y las estrategias analíticas más apropiadas para su análisis (KAPLAN, 1983; ZAVALETA, 1990; LACLAU, 1991; TAPIA, 2009 y 2006). En particular, el enfoque del Estado capitalista como condensación material de las relaciones de fuerza entre clases y fracciones de clases sociales (POULANTZAS, 1979), permitió minar la aparente unidad del Estado y sus aparatos, para dirigir la atención a la forma en que los conflictos sociales se traducen y reorientan selectivamente en las distintas disposiciones del mismo (CORTÉS, 2013). Por otra parte, el enfoque de la autonomía relativa del Estado capitalista, revitalizó problemáticas que iban más allá del reduccionismo economicista y el instrumentalismo, para pensar en cambio las complejidades inherentes a la relación entre el Estado y la sociedad civil en los distintos países:

\footnotetext{
(...) Poulantzas contribuye hasta cierto punto a que en la América Latina se fortalezcan los intentos de rescate y reivindicación del peso propio de lo político y de lo estatal. A las distinciones entre poder, política y Estado, y a la no identificación ni reducción del poder a las relaciones de clase ni al Estado, se agrega un Estado capitalista que se niega a ser instrumentado por clases externas a él, y que condiciona y determina la división social del trabajo, las relaciones económicas y las relaciones ideológico-políticas que en conjunto definen las clases (KAPLAN, 1983, p. 696).
}

Tanto el enfoque relacional del poder en el Estado, como su autonomía relativa respecto a las clases sociales (POULANTZAS, 1991a, 1991b y 1979), representaron un aporte que permitió ir más allá del determinismo económico en los debates latinoamericanos y dirigir la atención hacia las especificidades del Estado capitalista en la región. Si para exponentes del derivacionismo alemán resulta 
apropiado afirmar que lo económico no antecedió a lo político "ni teórica ni históricamente" (HIRSCH, 2005, p. 168), en el caso de las formaciones dependientes del hemisferio occidental esta premisa adquirió un peso aun mayor. En América Latina, el Estado habría sido el actor (...) "primordial en la sociedad, con una gama creciente de funciones y poderes y un papel decisivo en la reproducción del sistema o su eventual transformación" (KAPLAN, 1983, p. 677). En otros términos, si bien se reconoció durante estos años la dificultad de una teoría materialista del Estado para América Latina, debido en parte a los factores mencionados anteriormente sumando a ello los límites inherentes al economicismo y el instrumentalismo -, el trabajo de Poulantzas contribuyó a identificar no solo las especificidades del poder político, sino algunos de sus rasgos más relevantes en las formaciones dependientes.

Es así como Kaplan (1983) se aventuró a proponer, bajo lo que denominó como el Leviatán criollo, la categoría de "Estado neocapitalista tardío y dependiente". Con ello, buscó hacer alusión al carácter predominantemente productivo del Estado en la región así como a sus bajos niveles de autonomía relativa, que lo harían particularmente propenso al neofascismo. El Leviatán criollo, sería la expresión de un elevado grado de instrumentalización del poder estatal por parte de fracciones oligárquicas de las elites, con el fin de llevar a cabo las funciones extra-económicas necesarias para la articulación dependiente al mercado internacional, la existencia de monopolios (nacionales e internacionales) y la gran empresa. En América Latina, esta particular organización del Estado permitió crear las condiciones de (...) "instauración, estructuración, reproducción y crecimiento del neocapitalismo tardío" (KAPLAN, 1983, p. 700). De esta manera, advirtió tempranamente en contra de la hipótesis de una eventual reducción o suplantación del Estado por parte del mercado en un contexto neocapitalista y, por el contrario, avizoró que el papel de éste, debido en parte a su trayectoria histórica en la región, podría incrementarse a la hora de cumplir las funciones extra-económicas requeridas para la reproducción ampliada del capital. A propósito, destacó aspectos tales como la instauración de un ambiente institucional propicio para la atracción de inversiones extranjeras directas (eliminación de barreras arancelarias y jurídicas, entre otros) o la prestación de servicios de seguridad a compañías multinacionales.

Inspirado en el estatismo autoritario empleado por Poulantzas (1979) para describir el proceso de desmantelamiento del Estado Keynesiano de Bienestar en los países industriales, Kaplan (1983) sostuvo que las transformaciones del Estado a finales del siglo XX en América Latina no representarían tanto su reducción, como la reorientación de sus funciones y la acentuación de su carácter autoritario. Lejos de 
asistir al réquiem prematuro de las formas políticas de dominación en un contexto auto-regulado por el mercado, el Leviatán criollo tendería a consolidarse en aspectos tales como el predominio del ejecutivo sobre las demás ramas del poder político, el abuso de los medios masivos de comunicación, el vaciamiento de los mecanismos democráticos de participación y la creciente militarización de la sociedad como estrategia para reprimir el conflicto social y atraer inversiones privadas nacionales e internacionales (KAPLAN, 1983).

En un escenario de recesión económica e intervenciones norteamericanas, como el que tuvo lugar a finales de la década de 1970 en Latinoamérica (PANITCH, 2012; ROBINSON, 2015; GREEN y GRIFFITH, 2002), se perfilaba una crisis de los proyectos de Estado inspirados en el modelo de Industrialización por Sustitución de Importaciones (ISI) y el avance de proyectos neoliberales competitivos asociados con la apertura de los mercados. Si bien el desmantelamiento del modelo ISI significaba un nuevo momento para la autonomía relativa del Estado en la región, no representaba necesariamente una menor regulación en otros ámbitos. De un modo más preciso, era presumible que el Leviatán criollo experimentaría una nueva disposición de sus aparatos, en la cual aquellos relacionados con la articulación y reproducción del sistema capitalista global como los bancos centrales, ministerios de hacienda o agencias encargadas de conceder licencias extractivas incrementarían su papel, en ocasiones por fuera de todo control democrático.

En cuanto a la relación violencia-desarrollo, Kaplan (1983) anotó que la acentuación de los rasgos autoritarios del Estado podría hacer parte de la restructuración neocapitalista en América Latina, en contravía de una dependencia propiciada fundamentalmente por las relaciones económicas de inserción subordinada al mercado mundial, como la que planteó Marini (2008). Experiencias como el "fascismo sui generis del Cono Sur", que preludió la oleada de reformas que vendría en los ochenta, permitieron a Kaplan (1983, p. 707) prever la emergencia de nuevas formas de violencia funcionales a la reproducción global del capitalismo. Este aspecto es una de las razones que hacen de su análisis un vigoroso aporte para el entendimiento del Estado capitalista en la región y, por supuesto, otro motivo para dirigir la mirada al pensamiento fuerte latinoamericano, con el fin de lograr una mejor comprensión de las eventuales transformaciones del Leviatán criollo en un entorno de crisis del neoliberalismo (CORTÉS, 2013). 


\section{Contra la ruina de lo político: la ecuación social y la autonomia relativa del Estado capitalista en América Latina}

El debate que suscitó el problema del Estado capitalista en América Latina, encontró en Zavaleta otro interlocutor que llevó a cabo un trabajo particularmente provechoso en la materia. Parte de la obra realizada por Zavaleta (1990), quien siguió de cerca tanto el debate Miliband-Poulantzas (TARCUS, 1991) a propósito del Estado en las sociedades capitalistas, así como las contribuciones de la escuela alemana de la derivación, representó un paso más en el entendimiento de las especificidades del capitalismo y el Estado en la región. Coincidiendo con algunos de los aspectos anteriormente mencionados a propósito del pensamiento fuerte de América Latina, Zavaleta (1990) asumió que dado que varios determinantes del modo de producción capitalista no se encontraban presentes en la región al momento de la Conquista, este sería el resultado de la acción positiva del Estado.

En consecuencia, este no podría ser interpretado a partir de una lectura vulgar de la super-estructura, sino más bien como una fuerza auténticamente productiva (CORTÉS, 2013; OLIVER, 2006; TAPIA, 2006). Luego de reconocer con Aricó (2009) el carácter problemático de la unidad de América Latina, el cual dificulta una teoría del Estado capaz de englobar a toda la región en su heterogeneidad, Zavaleta (1990) admitió la pertinencia del legado de Marx (1992 y 1973) a la hora de pensar lo político en relación con las formas predominantes de la sociedad capitalista. Esto es, la disolución de las formas predominantes en la Edad Media y la abstracción formal del Estado respecto a la sociedad civil como rasgo constitutivo de lo político capitalista.

Acto seguido, sin embargo, aclaró que dicho razonamiento presenta un límite en el sentido de que permite observar la factualidad del Estado pero no su cualidad (ZAVALETA, 1990). Por lo tanto, la importancia de categorías históricas como "formación económico-social", "bloque histórico" y "superestructura" radica en que permiten observar la diversidad "de la historia del mundo" (ZAVALETA, 1990, p. 168-169). Si bien es cierto que los acontecimientos políticos e ideológicos entran en relación de un modo "diferido" y "remoto" con las expresiones inherentes al modo de producción, también lo es que dichos acontecimientos determinan el funcionamiento del acto productivo. Por lo tanto, las lecturas economicistas corren el riesgo de suprimir lo político en términos analíticos. Más que encontrar las leyes generales que dan forma al Estado capitalista en América Latina, es necesario analizar los distintos momentos en que se trasluce la primacía de lo político o viceversa: "lo que hay que definir son las fases de determinación lineal de la infraestructura económica sobre lo 
superestructural y los momentos (que son netos cuando existen) de primacía de lo político. Esto no habla de una cuestión de leyes sino de situaciones" (ZAVALETA, 1990, p. 169).

En el debate Miliband-Poulantzas (TARCUS, 1991; JESSOP, 2007), tomó distancia de ambos al sostener, por una parte, que el instrumentalismo de Miliband conducía a una taxonomía difusa que no permitía discernir con claridad el hecho de que el Estado puede encontrarse eventualmente en una situación instrumental (como la de Somoza en Nicaragua), sin que esto determine completamente su forma. Asimismo, señaló que la línea estructuralista adoptada por Poulantzas durante el debate, podría incurrir en lecturas estáticas que difuminan el acontecimiento político y el papel transformador de la agencia. No obstante, retomó de este último el enfoque de la autonomía relativa del Estado para construir un diálogo con Gramsci (2009) y llegar a una perspectiva conceptual más idónea a la hora de leer las realidades de América Latina: la ecuación social. Con dicho concepto, buscó identificar el grado de autonomía relativa del Estado en determinada formación social, tomando como variables las distintas posiciones de la escisión formal entre el Estado y la sociedad civil:

Por razones propias de cada caso, hay ecuaciones en las que la sociedad es más robusta y activa que el Estado, ecuaciones donde el Estado parece preexistir y dominar sobre la sociedad, al menos durante períodos determinados y sistemas donde hay una relación de conformidad o ajuste. Esta relación supone un movimiento y por eso es tan absurdo hacer clasificaciones finales sobre ello. La cualidad estatal, no estatal o intermedia de una instancia depende de su momento (ZAVALETA, 1990, p. 177).

Para este pensador, toda definición estática del Estado y sus aparatos representa la ruina de lo político. La ventaja de la ecuación social sobre el estructuralismo althusseriano, es que permite una definición dinámica de la relación entre los elementos originarios de lo político en las sociedades capitalistas: el Estado y la sociedad civil. En parte de Europa, por ejemplo, el ascenso de la burguesía permitió el desarrollo de condiciones favorables al capitalismo antes de que el Estado capitalista como tal se consolidara. En América Latina, el movimiento fue inverso. El Estado debió desarrollar las condiciones favorables para la implantación del capitalismo. Por lo mismo, una definición estática de la relación entre las formas sociales, conduciría a 
la parálisis histórica (ZAVALETA, 1990). Así las cosas, no es posible una teoría general del Estado sin pauperizar el análisis político, a menos que los elementos de dicha teoría se piensen de manera dinámica y se delimiten en la constitución histórica de cada Estado.

En este sentido, Zavaleta (1990) propuso un enfoque que contempló las categorías materialistas para el análisis del Estado, al tiempo que enfatizó en la necesidad de una perspectiva histórica que permitiera observar las expresiones diferenciadas de los acontecimientos políticos y productivos más allá de las formas homogéneas asociadas con la teoría de la dependencia. Para Oliver (2006), Zavaleta tomó la separación formal del Estado y la sociedad identificada por Marx (1992) como una teoría general que presenta, no obstante, formas específicas de materializarse en cada caso. Esto le permitió conservar un pie en la abstracción teórica y otro en la historia específica de cada formación social. En términos del mismo Zavaleta:

La ecuación o el bloque tiene entonces elementos verificables de historicidad y azar, no es una estructura predicha. Es una obra de los hombres materialmente determinados, algo que pudo haber sucedido de manera distinta a como sucedió. Como en todo modelo superestructural, podemos obtener algunas series causales o líneas de agregación pero en último término la teoría del Estado, si es algo, es la historia de cada Estado. Lo que importa, por tanto, es el recorrido de los hechos en la edificación de cada Estado (ZAVALETA, 1990, p. 180).

Por su parte, Tapia (2006) sostuvo que las pretensiones de Zavaleta no se ocuparon tanto de remplazar la teoría general del Estado, como de desarrollar perspectivas dinámicas e históricas de la misma. Resultaba preciso, desde este enfoque, estudiar los distintos casos de América Latina atendiendo tanto a los rasgos estructurales de la economía política, como al carácter abigarrado (unidad en la diversidad) y específico de las formaciones sociales del continente. Para ello, desarrolló conceptos y enfoques tales como el "momento constitutivo", con el cual aludió a las "razones originarias" o hechos fundantes que condujeron a determinada ecuación social a adquirir una forma y no otra (ZAVALETA, 1990, p. 180). Asimismo, propuso la "crisis como método", como una posibilidad de estudiar las formas políticas en situaciones de descomposición y recomposición con el fin de identificar el momento constitutivo (TAPIA, 2006; ANTEZANA, 2009). 
Entre otros, estos conceptos fueron intentos a través de los cuales procuró dar cuenta de la realidad de su país, Bolivia, y otros de América Latina como México y Argentina. En este sentido, Zavaleta (1990) no elaboró enfoques que sintetizan un conjunto de rasgos esenciales del Estado capitalista en la región, sino un haz de conceptos que apuntan a pensar la configuración compleja de la ecuación social en cada uno de los países. Más que una abstracción general que sería aplicada en cada caso, optó por categorías que le permitieran abordar lo concreto de cada país con el fin de analizar posteriormente las relaciones, las diferencias y lo que se comparte en común en el horizonte latinoamericano.

Debido a esto, su trabajo se considera una pieza clave para ir más allá de una teoría general del Estado en la región y construir marcos analíticos que contribuyan a la comprensión de las transformaciones políticas recientes (CORTÉS, 2013; TAPIA, 2006; OLIVER, 2006). De un modo más específico, el pensamiento de Zavaleta (1990) invita a interrogar la ecuación social que se ha cristalizado en cada Estado a partir de las reformas neoliberales de los años ochenta, al tiempo que advierte sobre los límites de una sociedad auto-regulada por las fuerzas del mercado. En lugar de asumir de manera incuestionada la utopía neoliberal, su obra permite plantear numerosos interrogantes, entre los cuales destacan: ¿Qué funciones productivas predominan en los Estados de América Latina luego de que tuvieran lugar las reformas políticas de la década de 1980 ? ¿Cómo se ha alterado la autonomía relativa del Estado en un escenario globalizado y qué implicaciones podría tener en la generación de crisis políticas inéditas en la región?

\section{El pensamiento fuerte de América Latina y el capitalismo global a debate}

Enfoques y categorías como los anteriormente mencionados, han inspirado debates importantes a la hora de pensar no solamente las especificidades del Estado en América Latina, sino también las transformaciones que en la materia ha representado la globalización neoliberal (CASTELLS y LASERNA, 1989; THWAITES y OUVIÑA, 2012; PANITCH, 2012; HEIGL, 2011; SOTELO y LAGNADO, 2017; PRADELLA, 2017; HOLLOWAY, 1993 y 1996; ROBINSON, 2015 y 2004). Sin embargo, antes de precisar algunos puntos de tensión al respecto, es necesario recordar que dentro de la corriente dependentista, hubo una heterogeneidad de puntos de vista sobre la relación de los Estados de la región con el mercado mundial, que hace difícil hablar de un cuerpo teórico completamente unificado. De hecho, hay 
perspectivas que aunque compartieron premisas centrales de dicha teoría, como la inserción subordinada de América Latina a los mercados internacionales o el carácter productivo del Estado en este proceso, fueron críticas de algunos de sus planteamientos.

Tal es el caso de Ocampo (1984), quien estuvo de acuerdo en que no es posible hacer un análisis riguroso del capitalismo en la región, sin tomar en consideración la articulación de sus economías al complejo del capitalismo mundial. Asimismo, reconoció que las desigualdades económicas en escala internacional son el producto de estructuras socio-económicas particulares y no simplemente un problema de "etapas" del desarrollo. También, coincidió en señalar que el origen histórico de las sociedades dependientes no es un proceso que se pueda pensar de manera aislada al de las sociedades "maduras", sino que constituye la otra cara de un mismo proceso histórico que se consolidó de manera desigual y diferenciada (OCAMPO, 1984, p. 24).

Sin embargo, consideró que no todas las periferias se articularon de igual forma al mercado mundial y, por lo tanto, adoptó el concepto de "periferias secundarias" para aludir a aquellas que fueron parcialmente dominadas e integradas al mercado internacional. El caso colombiano, constituye para este autor un ejemplo de dichas periferias secundarias, pues a pesar de que la dinámica de la economía de este país ha dependido en buena medida del sector externo, su articulación a los mercados internacionales ha sido históricamente precaria. Por otra parte, cuestionó a teóricos de la dependencia (GUNDER-FRANK, 1974; FURTADO, 1969) por asumir la formación de la economía mundial como un proceso temprano en la historia del capitalismo mercantil, el cual habría permitido la articulación de América Latina a la economía internacional desde la Conquista. Para Ocampo, tanto la consolidación del mercado mundial como la inserción de América Latina, fue un proceso complejo que tomó bastante tiempo y estuvo lleno de vaivenes (OCAMPO y BÉRTOLA, 2013). Por lo mismo, consideró que los conceptos de la teoría de la dependencia son funcionales ( $\mathrm{y}$ de manera no del todo precisa debido a su rigidez) a momentos de mayor consolidación del mercado mundial, e interrogó el hecho de:

...aplicar el concepto de la economía mundial, tal como lo conocemos hoy en día, a etapas anteriores del desarrollo del capitalismo. Aunque es cierto que el capitalismo tuvo un "mercado mundial" en todas las etapas de su desarrollo, también es cierto que por largos períodos históricos su alcance fue muy limitado (OCAMPO, 1984, p. 23). 
Por otra parte, cuestionó la primacía de los factores externos a la hora de explicar el desarrollo diferenciado de las periferias, lo que podría incidir en formas homogeneizantes de comprender una región de la complejidad y diversidad de América Latina. De allí su llamado a considerar también factores internos como la geografía o la composición social y política de cada país: "la forma de articulación con la economía mundial y las relaciones económicas internas constituyen dos aspectos mutuamente determinantes de una misma totalidad social" (OCAMPO, 1984, p. 59). Sin embargo, su trabajo se ha centrado más en los procesos de articulación económica al mercado mundial por parte de América Latina y Colombia en particular, que en el análisis de los procesos políticos que han conformado al Estado en la región. Lo cual no significa que desconozca el carácter productivo de los acontecimientos políticos en el desarrollo capitalista en Latinoamérica, sino que su foco de atención se dirigió a otros niveles del proceso.

Diferencias como las mencionadas, permiten observar los matices que se dieron al interior de la corriente dependentista y el pensamiento fuerte latinoamericano. Por lo tanto, las críticas que se abordarán al respecto, más que a un cuerpo doctrinario tomado en su conjunto, se centrarán en aspectos específicos, sin pretender reducir la pluralidad de puntos de vista mencionados a un único enfoque. Aclarado esto, se destacan las objeciones de Holloway (1993 y 1996) - quien fuera partícipe del debate alemán de la derivación durante sus inicios - a propósito de los límites de una forma rígida de comprender la polaridad Norte-Sur planteada por Marini (2008), así como la exterioridad Estado nacional-capital internacional en un contexto globalizado. Si bien coincidió en que la globalización no supone el desvanecimiento de los Estados nacionales, adujo que en el nuevo escenario no basta con hacer referencia a las presiones que los organismos multilaterales ejercieron sobre estos desde afuera para comprender sus más recientes transformaciones. Por el contrario, la comprensión de la globalización neoliberal pasa por disolver la aparente unidad del Estado y dirigir la atención a la manera en que fuerzas nacionales e internacionales se han articulado a la hora de crear un contexto de interacción global entre el trabajo y el capital.

Para Holloway (1993 y 1996), si bien la corriente dependentista logró plantear problemáticas relevantes respecto a la especificidad de los Estados capitalistas periféricos y la integración de los mismos a un sistema mundial, puede incurrir en perspectivas estadocéntricas que ofrecen una mirada estática de los Estados y las clases sociales tanto del centro como de la periferia. La condición de Estado dependiente o 
no dependiente no solo es para este autor siempre relativa sino que además, visto en términos del capital global, todos los Estados dependen de su capacidad para atraer, retener y reproducir el capital flotante en escalas internacionales hacia el interior de sus fronteras. Asimismo, al no hacer precisión sobre el hecho de que no hay una exterioridad real entre el Estado nacional y el capital global, las reformas neoliberales pueden verse reducidas a simples imposiciones externas, sin que se ofrezcan alternativas que expliquen la dinámica exterior/interior que se conformó durante las reformas de finales del siglo XX (HOLLOWAY, 1993 y 1996).

Desde este enfoque, la polaridad que ofrece la globalización neoliberal no es la misma que hubo en momentos previos al desarrollo del mercado global, esto es, entre países del centro industrial y países periféricos o dependientes como, entre otros, sostuvo Marini (2008). La nueva polaridad que se ha venido cristalizando desde finales del siglo XX es, para Holloway (1993), entre el capital global y el trabajo global. Esto quiere decir que no hay propiamente una competencia entre capitales nacionales del centro y la periferia por retener un capital global inmóvil:

El punto de partida en la discusión tiene que ser no la inmovilidad del capital sino su movilidad. En tanto que la existencia de cualquier Estado nacional depende no solamente de la reproducción del capitalismo mundial, sino de la reproducción del capitalismo dentro de sus fronteras, el Estado tiene que tratar de atraer y, una vez atraído, de inmovilizar al capital dentro de su territorio. La lucha competitiva entre los Estados nacionales no es una lucha entre capitales nacionales, sino una lucha entre los Estados para atraer y/o retener una parte del capital mundial y por lo tanto una parte de la plusvalía global. Para conseguir eso, el Estado nacional tiene que tratar de asegurar condiciones favorables para la reproducción del capital dentro de sus fronteras, proveyendo la infraestructura, el orden público, la educación y la regulación de la fuerza de trabajo, etcétera. Además tiene que dar apoyo internacional - a través de la política comercial, política monetaria, intervención militar, etcétera - al capital operando dentro de sus fronteras, generalmente haciendo caso omiso de la nacionalidad de los propietarios legales de ese capital (HOLLOWAY, 1993, p. 19).

Una de las consecuencias de la nueva relación Estado-capital global, no sería tanto la delegación progresiva de funciones del Estado en el mercado hasta su posterior debilitamiento, sino el ahuecamiento de las democracias y la cada vez mayor subordinación de los intereses de la población nacional ante los del capital global. En este punto, podría hablarse de un grado de coincidencia con trabajos como los de 
Zavaleta (1990), Kaplan (1983) o Lechner (1980), quienes anunciaron una mayor instrumentalización del Estado a medida que avanzaba la internacionalización del capital. Para Holloway, este proceso consistió en la implementación de reformas que permitieron a los Estados nacionales depender menos de los mercados internos y más de la atracción de flujos internacionales de capital. No obstante, la internacionalización competitiva por parte de cada uno de los Estados de Latinoamérica, es un fenómeno que desde este punto de vista requiere de estudios que permitan observar tanto los rasgos específicos como las tendencias generales.

En esta dirección, apuntan trabajos recientes de Robinson (2015 y 2004) sobre América Latina, quien junto con Gill (1995) y Cox (2013) puede considerarse como uno de los representantes de la escuela del capitalismo global, la cual sostiene que a finales del siglo XX se conformó un nuevo momento en la historia del capitalismo, caracterizado a grandes rasgos por la hegemonía de la Clase Capitalista Transnacional (CCT), la deslocalización de la producción y la emergencia de un Estado Capitalista Transnacional (ECT) (ROBINSON, 2007). Tomando en consideración algunos elementos de la teoría de la dependencia, tales como las diferentes formas de inserción al mercado mundial entre el centro y las periferias, sostuvo que el nuevo escenario propuesto por el capitalismo global no puede resumirse a un ciclo de capitales extranjeros apropiándose de los nacionales. Más bien, consistiría en una compleja imbricación, en la cual los capitales nacionales han recurrido al Estado para atraer a sus territorios capitales extranjeros con alta movilidad, fusionarse con ellos y eventualmente hacer alianzas regionales para competir.

No se trata ahora de una burguesía compradora que, actuando como socio menor, vende solo materia prima, en lo que sería el ciclo dependiente del capital descrito por Marini (2008). Hay también un considerable desarrollo industrial en las periferias asociado a la deslocalización de la producción, procesos complejos de inversión regional que se encuentran orientados a un funcionamiento transnacional de la economía, el cual incluso ha permitido llevar flujos de Inversión Extranjera Directa del Sur al Norte global. Más que de un enfrentamiento entre capitales nacionales contra las compañías transnacionales en la periferia, se trata de la articulación entre fracciones de las élites de América Latina con circuitos de la Clase Capitalista Transnacional (van der PIJL, 1998) que, entre otras cosas, ha transformado al Estado en la región (ROBINSON, 2015). 
Es necesario, también desde este enfoque, repensar el carácter fijo de la categoría Norte-Sur y el ciclo dependiente del capital, según el cual el Norte explota al Sur a través de una división internacional del trabajo, consistente en el envío desde el Sur de materias primas y mano de obra barata. Aunque este patrón, profundizado por Marini (2008) como herencia de la conformación del mercado mundial, continúa operando a grandes rasgos, ha tenido desde este punto de vista variaciones importantes durante las últimas décadas del siglo XX. Ahora el Norte también envía al Sur alimentos producidos con mano de obra barata, sumándole a dicho proceso el uso de tecnología avanzada en una combinación atípica de plusvalía intensiva y extensiva. Los nuevos complejos agroindustriales en el Sur se han californizado, aludiendo con ello a la combinación de mano de obra barata y tecnología avanzada. Asimismo, el producto de estos complejos no solo se dirige a los países del Norte sino que fluye a través del mundo gracias en parte a las nuevas tecnologías del transporte y la comunicación. En suma, los capitales nacionales y extranjeros no se comportarían propiamente como rivales, sino que se articulan de manera transversal, en un proceso que requiere de una cada vez mayor instrumentalización del poder del Estado.

Para analizar este nuevo escenario, es indispensable ir más allá del Estadonación centrismo y atender el impacto que generan los procesos transnacionales. Por lo tanto, además de replantear categorías legadas por el pensamiento fuerte en la región, esta escuela pretende desarrollar estrategias metodológicas que vayan más allá de la relación entre Estados nacionales e incorporen las nuevas relaciones que trae el capitalismo global. En especial las diversas formas de articulación en torno al liderazgo moral y político de la Clase Capitalista Transnacional (ROBINSON, 2007). Destacan en este sentido, estrategias que indaguen la nueva dialéctica exteriorinterior, con el fin de comprender las formas en que las compañías transnacionales y organismos multilaterales se han convertido en actores políticos con capacidad de incidencia en el diseño de políticas públicas, la liberalización de los mercados, la integración comercial y la privatización, especialmente a partir de la década de 1990.

Más allá de una imposición proveniente desde afuera, las reformas neoliberales consistirían en un haz de mecanismos que ha reorganizado a los Estados nacionales desde adentro, con procesos que incluyen el ascenso de una élite tecnocrática (tecno-pols) que aplica la racionalidad capitalista en el diseño de las políticas y privilegia una escala de regulación global a una nacional (ROBINSON, 2015). Los mecanismos van desde la reproducción e incorporación de nuevos imaginarios políticos y económicos, hasta la financiación de campañas y partidos por parte de capitales privados, la ubicación de miembros favorables a las compañías en 
cargos estratégicos de los aparatos del Estado y los partidos, creación de institutos de planeación y Think-Tanks que elaboran propuestas, programas y planes de desarrollo.

Esta transformación del Estado, cuya principal característica de acuerdo con Robinson (2007) radica en una presencia cada vez más estratégica de fracciones transnacionalizadas de las elites nacionales (asociadas fundamentalmente con los capitales financieros y comerciales), conduce no tanto a la disolución del Estado nación como a su articulación con un aparato de Estado transnacional. Dicho aparato, aunque cuenta con redes alrededor del mundo, tiende a centralizarse en los Estados Unidos con el objetivo de coordinar desde allí la operación conjunta del capitalismo. El Estado Capitalista Transnacional (ECT), sería entonces el resultado de una serie de aparatos y circuitos ideológicos, políticos y económicos reproducidos por la Clase Capitalista Transnacional, con el propósito de incidir en el diseño de cada Estado nacional y propiciar la deslocalización de la producción, la permanencia de los patrones globales de acumulación y la gerencia centralizada de las operaciones globales (ROBINSON, 2007).

Sin embargo, esta apreciación ha sido cuestionada por no precisar lo suficiente el papel de cada Estado nacional en el supuesto ECT, así como el lugar, todavía vigente, del sistema internacional de Estados en la conformación del mercado global (HIRSCH y KANNANKULAM, 2011; HIRSCH y WISSEL, 2011; GERSTENBERGER, 2011; PICCIOTO, 2011). Para Hirsch y Wissel (2011), más que la integración de un aparato de Estado transnacional, se trataría de la reorganización del sistema internacional de Estados a través de la internacionalización de los proyectos políticos y los aparatos estatales desde un enfoque competitivo. Aspectos como la disposición de ambientes legales e institucionales, la construcción de infraestructura o los servicios de seguridad, no pueden ser provistos por el mercado o agencias externas a los Estados individuales.

Para esta perspectiva, heredera de la escuela alemana de la derivación, podría resultar más pertinente un enfoque como el de Kaplan (1983), el cual resalta el carácter productivo de los Estados dependientes en el neocapitalismo a través de la acentuación de sus funciones extra-económicas. Más que la integración de los Estados nacionales en favor de un aparato de Estado transnacional (ROBINSON, 2007), se trataría de la internacionalización de las escalas de regulación a través de proyectos políticos competitivos, que ofrecerían al mercado internacional el ambiente institucional necesario para su operación. Por supuesto, esto no descarta un elevado grado de instrumentalización del Estado por parte de las elites nacionales e 
internacionales que, al comprometer los niveles de autonomía relativa, puede redundar en escenarios propicios a crisis violentas (HIRSCH y WISSEL, 2011). De manera que aunque la escuela del capitalismo global tiene el mérito de plantear un debate novedoso, no ha generado el consenso suficiente a la hora de descartar algunas de las categorías desarrolladas en América Latina con el propósito de pensar el Estado y el capitalismo.

No obstante, reconocer debates como los propuestos por Robinson (2015 y 2007) y Holloway (1993 y 1996), permite observar la pertinencia de retomar con nuevos aires aspectos del pensamiento fuerte latinoamericano, atendiendo especialmente a los impactos que sobre este pueden presentar la alta movilidad del capital y la deslocalización de la producción que tuvo lugar a finales del siglo XX. En este nuevo escenario, el presente artículo sostiene, sin embargo, que algunas de las reflexiones planteadas por dicha corriente, pueden todavía arrojar alternativas teóricas y metodológicas para el análisis del Estado capitalista en América Latina. Destacan, en este sentido, tres de sus aspectos seminales: a) el carácter altamente productivo de los Estados en la región, el cual conlleva a una aproximación metodológica por fuera del dualismo Estado-mercado a la hora de comprender las formas de articulación entre elites nacionales y transnacionales; b) las formas desiguales de inserción al mercado mundial que, aunque han presentado cambios, siguen operando de manera diferenciada en las formaciones sociales en desarrollo e industrializadas, y, c) las variaciones en los niveles de autonomía relativa del Estado, las cuales permiten hacer énfasis en su transformación y la acentuación de su carácter autoritario.

En primer lugar, las reformas neoliberales para América Latina no han representado un retroceso del Estado ante el mercado, ni la disolución de aquel en un ECT con sede en EE.UU. (a pesar de la creciente participación en el diseño de políticas por parte de organismos multilaterales y otros actores transnacionales). Se ha tratado más bien de la incorporación de una lógica mercantil en el diseño institucional y los proyectos políticos, con el propósito de producir las condiciones necesarias para una articulación subordinada al mercado global. En este punto, hay un grado de coincidencia entre pensadores como Kaplan (1983) y trabajos recientes como el de Hirsch y Wissel (2011), donde se descarta la eliminación prematura de la forma política del capitalismo y el sistema de Estados, para dar lugar a los rasgos generales de su transformación contemporánea bajo la categoría de Estados internacionalizados-competitivos.

Por otra parte, si bien las observaciones mencionadas a propósito de la industrialización del Sur y la creciente polaridad entre el trabajo y el salario global no 
carecen de fundamentos (HOLLOWAY, 1993 y 1996; ROBINSON, 2015), los patrones de inserción subordinada fueron incluso identificados por parte de organismos como el FMI y el Banco Mundial durante la década de las reformas. Dichos organismos, advirtieron que mientras los países de regiones como América Latina avanzaban en la implementación de ajustes estructurales y el desmantelamiento del modelo de Industrialización por Sustitución de Importaciones, los países industriales no solo mantenían esquemas proteccionistas, sino que además diseñaban nuevas barreras no arancelarias que amenazaban con profundizar formas desiguales de inserción al mercado mundial (IMF, 1983, 1984 y 1986; WORLDBANK, 1983, 1985 y 1986). Esto nos permite observar que, a pesar de las tendencias anteriormente enunciadas, las polaridades Norte-Sur no se han diluido por completo y, casos como el colombiano, permiten asociar el desmantelamiento de las estrategias de industrialización dirigidas por el Estado, con un crecimiento notable de las industrias extractivas, las cuales han propiciado una reprimarización de las economías y, por tanto, nuevas formas de articulación dependiente a los mercados globales (BONILLA, 2011; OCAMPO y BÉRTOLA, 2013).

En cuanto a la creciente instrumentalización de los Estados por parte de elites nacionales e internacionales y, por ende, sus bajos niveles de autonomía relativa, también cobran pertinencia aspectos asociados con las reflexiones de Kaplan (1983), Tapia (2009) o Zavaleta, (1990). La internacionalización de los proyectos políticos (JESSOP, 2015), no se encuentra relacionada propiamente con una delegación de las soberanías nacionales o el retroceso del Estado ante el mercado, sino más bien con el despliegue de estrategias de gobierno que incrementan las funciones extraeconómicas, esenciales para el flujo de los capitales transnacionales. La acentuación de los rasgos violentos y autoritarios, por supuesto, no se encuentra descartada en este nuevo escenario. Todo lo contrario, como bien lo señalaron Hirsch y Wissel (2011), el monopolio legítimo de la fuerza que reposa en los Estados individuales, sigue desempeñando un papel central que no puede ser asumido completamente por el mercado sin comprometer la forma política del capitalismo. De allí que las expresiones del fascismo autóctono, como las denominó Kaplan (1983), apunten a estar a la orden del día en un contexto globalizado, el cual presenta una compleja relación violencia-desarrollo.

A propósito de esto último, el caso colombiano provee un claro ejemplo del nuevo rostro del Leviatán criollo en la región. Sin la necesidad de incurrir en medidas de excepción prolongadas - como las dictaduras del Cono Sur -, dicho Estado ha 
desplegado estrategias de gestión público-privadas de la violencia, las cuales han sido altamente funcionales a los capitales transnacionales, pese a que un ambiente convulso pueda elevar de manera eventual los costos de transacción (RICHANI, 2013). Especialmente, los sectores primarios de extracción minero-energética o agro bussiness, han prosperado en un entorno que parece indicar que, bajo el manto novedoso del capitalismo global, todavía se asoma el "pecado original” de la conformación del mercado mundial: la acumulación primitiva (BALLVÉ, 2013; HRISTOV, 2014; ZELIK, 2015)2.

De manera que ante debates como los propuestos por Holloway (1993 y 1996) o Robinson (2015), los aportes de la corriente del pensamiento fuerte que han sido señalados, presentan todavía vigencia a la hora de abordar las nuevas transformaciones del Estado capitalista en América Latina. No obstante, la agenda de estudios puede verse enriquecida al considerar el impacto de factores asociados con el nuevo momento del capitalismo y el carácter cada vez más activo de los organismos multilaterales y actores transnacionales en el diseño de las políticas en los países en desarrollo. No se desconoce, por supuesto, que la así llamada escuela del capitalismo global, tiene todavía mucho que aportar a la comprensión de las nuevas realidades latinoamericanas, aunque sea todavía necesario precisar en mayor detalle la forma en que comprende la conformación de un Estado Capitalista Transnacional y el lugar que allí ocuparían tanto los Estados nacionales como el sistema internacional de Estados.

\section{Conclusión}

Los desafíos planteados por la globalización a la hora de comprender el Estado capitalista en la región, en consecuencia, son numerosos. La ola de reformas que tuvo lugar entre los años 1980s y 1990s, introdujo cambios tan profundos y generalizados, que ha sido denominada como una auténtica revolución silenciosa (GREEN y GRIFFITH, 2002). De acuerdo con Cortés (2013), el momento actual se muestra propicio para retomar los elementos centrales del pensamiento fuerte en

\footnotetext{
${ }^{2}$ La alusión a este caso se sustenta en la aparente paradoja que supone el recrudecimiento de un conflicto interno que en sus más de 5 décadas de duración ha dejado aproximadamente 200.000 víctimas fatales, al tiempo que la Inversión Extranjera Directa ha mostrado un crecimiento de niveles históricos en sectores como el minero-energético y el agroubussiness. De allí que los estudios anteriormente citados, recurran a dicho caso con el fin de observar la compleja relación entre las estrategias de acumulación y desarrollo del capitalismo global y las nuevas formas de violencia asociadas con la acentuación de las funciones extra-económicas del Estado capitalista dependiente.
} 
América Latina, especialmente a la hora de comprender aquello que Kaplan (1983) denominó como el Leviatán criollo, en alusión al papel productivo del Estado neocapitalista tardío y dependiente. No obstante, consideramos válido el siguiente interrogante: ¿Podemos retomar esta corriente de la misma manera en que se presentó a partir de los años 70s del siglo XX? Si bien algunos de los conceptos y enfoques que el pensamiento fuerte ha legado en la materia tienen todavía mucho que aportar, es menester proseguir en la comprensión de los rasgos específicos del capitalismo global, con el fin de interrogar de manera menos rígida categorías como Norte-Sur global y Estado nacional-capital internacional. En efecto, si como sostuvo Zavaleta (1990), una teoría general del Estado capitalista en América Latina solo puede ser concebida en términos históricos, la comprensión de los rasgos específicos del nuevo momento del capitalismo y el modo en que se ha articulado la región a partir de las reformas de comienzos de los años 1990s, es un aspecto central para el análisis político.

Por otra parte, un elemento central de la teoría dependentista propuesta por Marini (2008) ha sido cuestionado por Holloway (1993 y 1996) y Robinson (2015), quienes abrieron un campo de debate al plantear que en el nuevo contexto global todos los Estados dependen de la atracción y retención de flujos internacionales de capital en su territorio. Sin embargo, es necesario reconocer que las formas en que estos dependen son heterogéneas: hay selectividades estratégicas que se expresan todavía en las formas de dependencia, internacionalización del Estado y acceso diferenciado a los mercados del capital. La expresión de dichas selectividades, permite cuestionar la idea de que la globalización represente esencialmente una mayor integración de mercados y fuerzas en los mismos términos para todos los países. Si bien es posible observar una integración de procesos, flujos (migracionales y de capitales) y agencias transnacionales, la persistencia de formas de acumulación a escala global que distancian y disgregan cada vez más a los países industrializados de los países en desarrollo - expresadas en la gran divergencia que se incrementó con la globalización neoliberal (OCAMPO y BÉRTOLA, 2013) -, constituye un indicio de que las formas económicas, jurídicas y políticas del capitalismo global presentan especificidades que no pueden ser abarcadas por una teoría general sin desmedro de una perspectiva histórica, la cual constituye una de las bases de la crítica materialista.

Aunque en un contexto de crisis o recomposición del modelo neoliberal no es posible predecir las transformaciones estatales en América Latina, se pueden avizorar algunos aspectos que de manera eventual enriquecerían la agenda de estudios y que, por el momento histórico propio de la corriente del pensamiento fuerte, quizás no se 
presentaron con la claridad que al día de hoy es posible observar. Uno de los atributos centrales de la dependencia, de acuerdo con Marini (2008), radica en una articulación subordinada al mercado mundial por parte de las formaciones sociales periféricas, la cual prescinde de la ocupación militar directa que caracterizó a la experiencia colonial. No obstante, la compleja relación violencia-desarrollo que ha sido estudiada en casos como el colombiano (BALLVÉ, 2013; HRISTOV, 2014; ZELIK, 2015), advierte sobre los nuevos roles que pueden desempeñar los Estados latinoamericanos y las fuerzas transnacionales en una vieja práctica del mercado mundial: la acumulación primitiva.

Finalmente, más allá de los limitantes internos a la expansión global del capitalismo, ligados a expresiones sociales de lucha y resistencia, aparece un nuevo factor que debe ser tenido en cuenta. El desmantelamiento de las estrategias de industrialización dirigidas por el Estado, así como la emergencia de los proyectos políticos competitivos que orientaron las reformas neoliberales entre los años 1980s y 1990s, profundizaron formas extractivas de articulación al sector externo cuyo impacto sobre el medio ambiente, la composición del territorio y las poblaciones que lo habitan están todavía por dimensionar (OCAMPO y BÉRTOLA, 2013). Bástenos en este punto con advertir que el Leviatán criollo no solamente ha desplegado formas autoritarias y violentas con respecto a las luchas que lo han resistido, sino que en su afán por aprovechar las ventajas competitivas y atraer flujos internacionales de Inversión Extranjera Directa, amenaza también con infringir daños irreparables en los ecosistemas del continente, especialmente derivados del impacto de las industrias extractivas en el sector minero-energético: ¿Será la naturaleza - base material indiscutible de la riqueza - un factor a tener en cuenta durante los próximos años entre los limitantes a la instrumentalización de los Estados capitalistas en la región?

Juan Camilo Arias é Mestre em Estudos Políticos, pela Universidad Nacional de Colombia, Medellin. Doutorando em Ciências Humanas e Sociais, pela Universidad Nacional de Colombia, Medellin. E-mail: jcariasm1@gmail.com. 


\section{Referências}

ANTEZANA, Luis. La crisis como método en René Zavaleta Mercado. Ecuador Debate, n. 77, p. 107-124, 2009. Disponible en: <http://repositorio.flacsoandes.edu.ec/handle/10469/4339>. Acceso en: 6 nov. 2018.

ARICÓ, José. Marx y América Latina. México: Fondo de Cultura Económica, 2009.

BALLVÉ, Teo. Grassroots masquerades: Development, paramilitaries, and land laundering in Colombia. Geoforum, n. 50, p. 62-75, 2013. Disponible en: <https://www.academia.edu/4030170/ Grassroots_Masquerades_Development_Paramilitaries_and_Land_Laundering_in_Colombia>. Acceso en: 6 nov. 2018.

BARROW, Clyde. The Marx Problem in Marxian State Theory. Science \& Society, v. 64, n. 1, p. 87118, 2000. Disponible en: <https://www.jstor.org/stable/40403826?seq=1\#page_scan_ tab_contents>. Acceso en: 6 nov. 2018.

BONILLA, Ricardo. Apertura y reprimarización de la economía colombiana. Nueva Sociedad, n. 231, p. 46-65, 2011. Disponible en: <http://nuso.org/media/articles/downloads/3752_1.pdfs. Acceso en: 6 nov. 2018.

CASTELLS, Manuel; LASERNA, Roberto. La nueva dependencia. Cambio tecnológico y reestructuración socioeconómica en Latinoamérica. David y Goliath, v. 18, n. 55, p. 2-16, 1989. Disponible en: <http://biblioteca.clacso.edu.ar/ar/libros/historico/dyg/55.pdfs. Acceso en: 6 nov. 2018.

CORTÉS, Martín. El Leviatán criollo. Elementos para el análisis de la especificidad del Estado en América Latina. In: THWAITES, Mabel (Ed.). El Estado en América Latina: continuidades y rupturas. Santiago de Chile: CLACSO, 2013, p. 93-117.

COX, Robert. Fuerzas sociales, estados y órdenes mundiales: Más allá de la teoría de Relaciones Internacionales. Relaciones Internacionales, n. 24, p. 129-162, 2013. Disponible en: <https://repositorio.uam.es/handle/10486/677391>. Acceso en: 6 nov. 2018.

FALETO, Enzo. La especificidad del Estado en América Latina. Revista CEPAL, n. 38, p. 69-87, 1989. Disponible en: <https://repositorio.cepal.org/handle/11362/11740>. Acceso en: 6 nov. 2018.

FOUCAULT, Michell. Power/Knowledge: Selected Interviews and Other Writings 1972-1977. New York: Colin Gordon, 1980.

FURTADO, Celso. La economía latinoamericana desde la Conquista hasta la Revolución Cubana. México: Siglo Veintiuno, 1969.

GERSTENBERGER, Heide. The Historical Constitution of the Political Forms of Capitalism. Antipode, v. 43, n. 1, p. 60-86, 2011. Disponible en: <https://onlinelibrary.wiley.com/doi/abs/ 10.1111/j.1467-8330.2010.00811.x>. Acceso en: 6 nov. 2018.

GILL, Stephen. Globalisation, Market Civilisation and Disciplinary Neoliberalism. Millennium: Journal of International Studies, v. 24, n. 3, p. 399-423, 1995. Disponible en: <http://journals.sagepub.com/doi/10.1177/03058298950240030801>. Acceso en: 6 nov. 2018.

GRAMSCI, A. The Gramsci Reader: Selected Writings 1916-1935. New York: University Press, 2009.

GREEN, Duncan; GRIFFITH, Matthew. Globalization and its Discontents. International Affairs (Royal Institute of International Affairs), v. 78, n. 1, p. 49-68, 2002. Disponible en: <https://www.jstor.org/stable/3095974?seq=1\#page_scan_tab_contents>. Acceso en: 6 nov. 2018. 
GUNDER-FRANK, André. Capitalismo y subdesarrollo en América Latina. Buenos Aires: Siglo Veintiuno Editores, 1974.

HEGEL, Firedrich. Philosophy of Right. Kitchener: Batoche Books Limited, 2001.

HEGEL, Friedrich. Political Writings. Cambridgeshire: Cambridge University Press, 2004.

HEIGL, Miriam. Social Conflict and Competing State Projects in the Semi-Periphery: A StrategicRelational Analysis of the Transformation of the Mexican State into an Internationalized Competition State. Antipode, v. 43, n. 1, p. 129-148, 2011. Disponible en: $<$ https://onlinelibrary.wiley.com/doi/abs/10.1111/j.1467-8330.2010.00814.x>. Acceso en: 6 nov. 2018.

HEINRICH, Michael. An Introduction to the Three Volumes of Karl Marx's Capital. New York: Monthly Review Press, 2004.

HIRSCH, Joachim; KANNANKULAM, John. The Spaces of Capital: The Political Form of Capitalism and the Internationalization of the State. Antipode, v. 43, n. 1, p. 12-37, 2011. Disponible en: <https://onlinelibrary.wiley.com/doi/abs/10.1111/j.1467-8330.2010.00809.x>. Acceso: 6 nov. 2018.

HIRSCH, Joachim; WISSEL, Jens. The transformation of contemporary capitalism and the concept of a transnational capitalist class: a critical review in neo-poulantzian perspective. Studies in Political Economy, v. 88, n. 1, p. 7-33, 2011. Disponible en: <https://www.tandfonline.com/doi/abs/ 10.1080/19187033.2011.11675007?journalCode=rsor20>. Acceso en: 6 nov. 2018.

HIRSCH, Joachim. ¿Qué significa Estado? Reflexiones acerca de la teoría del Estado Capitalista. Curitiba, n. 24, p. 165-175, 2005. Disponible en: <http://www.redalyc.org/pdf/238/23802411.pdf>. Acceso en: 6 nov. 2018.

HOLLOWAY, John. La reforma del Estado: capital global y Estado nacional. Perfiles Latinoamericanos, n. 1, p. 7-32, 1993. Disponible en: <http://www.redalyc.org/pdf/ 115/11500102.pdf>. Acceso en: 6 nov. 2018.

HOLLOWAY, John. Global Capital and the National State. In: HOLLOWAY, John; BONEFIELD, Werner (Ed.). Global Capital, National State and the Politics of Money. London: Macmillan Press LTDA, 1996, p. 116-141.

HRISTOV, Jazmin. Paramilitarism and Neoliberalism. Violent Systems of Capital Accumulation in Colombia and Beyond. London: Pluto Press, 2014.

IMF. Annual Report, 1983. Disponible en: <https:/www.imf.org/external/pubs/ft/ar/archive/pdf/ ar1983.pdfs. Acceso en: 6 nov. 2018.

IMF. Annual Report, 1984. Disponible en: <https:/www.imf.org/external/pubs/ft/ar/archive/pdf/ ar1984.pdf>. Acceso en: 6 nov. 2018.

IMF. Annual Report, 1986. Disponible en: <https:/www.imf.org/external/pubs/ft/ar/archive/pdf/ ar1986.pdf>. Acceso en: 6 nov. 2018

JESSOP, Bob. Dialogue of the deaf: reflections on the Poulantzas-Miliband debate. In: BARROW, Clyde; BURnhaM, Peter; WeTHERLY, Paul (Eds.). Class, Power and the State in Capitalist Society: Essays on Ralph Miliband. Basingstoke: Palgrave. 2007, p. 132-157,

JESSOP, Bob. Poulantzas's State, Power, Socialism as a Modern Classic. In: GALLAS, Alexander; BRETTHAUER, Lars; KANNANKULAM, John; STUZLE, Ingo (Eds.). Reading Poulantzas. London: Merlin, 2011, p. 42- 55.

JESSOP, Bob. The State. Past, Present, Future. Cambridge: Polity Press, 2015. 
KAPLAN, Marcos. La teoría del Estado en la América Latina contemporánea: El caso del marxismo. El Trimestre Económico, v. 50, n. 198(2), p. 677-711, 1983. Disponible en: <https://www.jstor.org/stable/23395699?seq=1\#page_scan_tab_contents>. Acceso en: 6 nov. 2018.

LACLAU, Ernesto. La especificidad de lo político. In: TARCUS, Horacio (Ed.). Debates sobre el Estado capitalista. Estado y clase dominante. Buenos Aires: Imago Mundo, 1991, p. 121-153.

LECHNER, Norbert. La crisis del Estado en América Latina. Caracas: El Cid Editor, 1977.

LECHNER, Norbert. Estado y Política en América Latina. In: Conferencia dictada en la Universidad Internacional Menéndez Pelayo, España. Flacso-Documento de Trabajo, 1980.

MARINI, Ruy Mauro. América Latina, dependencia y globalización. MARTINS, Carlos (Comp.). Bogotá: CLACSO y Siglo del Hombre Editores, 2008.

MARX, Karl. El Capital. Crítica de la Economía Política. México: Fondo de Cultura Económica, 1973.

MARX, Karl. Critique of Hegel's Doctrine of the State. In: Marx, Karl.: Early Writings. London: Penguin Books-New Left Review, 1992. p. 8-57.

MEDINA, Victor. El Estado capitalista en América Latina, ¿potencial factor de cambio o mero instrumento de dominación? Izquierdas, n. 31, p. 219-234, 2016. Disponible en: <http://www.redalyc.org/pdf/3601/360149583010.pdf>. Acceso en: 6 nov. 2018.

OCAMPO, José; BÉRTOLA, Luis. El desarrollo económico de América Latina desde la Independencia. México: Fondo de Cultura Económica, 2013.

OCAMPO, José. Colombia y la economía mundial, 1830-1910. Bogotá: Siglo XXI Editores, 1984.

OLIVER, Lucio. René Zavaleta ante la especificidad latinoamericana del Estado y la política. In: AGUILUZ, Maya; DE LOS RIOS, Norma (Eds.). René Zavaleta Mercado. Ensayos, testimonios y re-visiones. Buenos Aires: Flacso, 2006, p. 213-225.

PANITCH, Leo. The Impoverishment of State Theory. Socialism and Democracy, v. 13. p. 19-35, 1999. Disponible en: <https:/www.tandfonline.com/doi/abs/10.1080/08854309908428242? journalCode $=$ csad20>. Acceso en: 6 nov. 2018.

PANITCH, Leo. American empire, capitalist crisis and the global south. Keynote speech to the Society for Socialist Studies, v. 8, n. 2. p. 1-12, 2012. Disponible en: <https://www.socialiststudies.com/index.php/sss/article/view/23542/17426>. Acceso en: 6 nov. 2018.

PICCIOTO, Sol. International Transformations of the Capitalist State. Antipode, v. 43, n. 1, p. 87107, 2011. Disponible en: <https://onlinelibrary.wiley.com/doi/abs/10.1111/j.14678330.2010.00812.x>. Acceso en: 6 nov. 2018.

POULANTZAS, Nikos. La internacionalización de las relaciones capitalistas y el Estado-Nación. Investigación Económica, v. 32, n. 127, p. 519-553, 1973. Disponible en: <https://www.jstor.org/ stable/42813615?seq=1\#page_scan_tab_contents>. Acceso en: 6 nov. 2018.

POULANTZAS, Nikos. The Crisis of the Dictatorships: Portugal, Spain, Greece. London: NLB, 1976.

POULANTZAS, Nikos. Estado, poder y socialismo. Madrid: Siglo XXI Editores, 1979.

POULANTZAS, Nikos. El problema del Estado capitalista. In: TARCUS, Horacio (Comp.). Debates sobre el Estado capitalista. Estado y clase dominante. Buenos Aires: Imago Mundo, 1991a. p. 71-91. 
POULANTZAS, Nikos. El Estado capitalista: una réplica a Miliband y Laclau. In: TARCUS, Horacio (Comp.). Debates sobre el Estado capitalista. Estado y clase dominante. Buenos Aires: Imago Mundo, 1991b, p. 153-185.

POULANTZAS, Nikos. Poder político y clases sociales en el Estado capitalista. México: Siglo XXI Editores, 2007.

PRADELLA, Lucía. Marx and the Global South: Connecting History and Value Theory. Sociology, v. 51, n. 1, p. 146-161, 2017. Disponible en: <http://journals.sagepub.com/doi/pdf/10.1177/ $0038038516661267>$. Acceso en: 6 nov. 2018.

PRADELLA, Lucía. Globalisation and the Critique of Political Economy. New insights from Marx's writings. Oxford: Routdledge, 2015.

RICHANI, Nazi. Systems of Violence, Second Edition: The Political Economy of War and Peace in Colombia. Albany: State University of New York Press, 2013.

ROBINSON, William. Global Crisis and Latin America. Bulletin of Latin American Research, v. 23, n. 2, p. 135-153, 2004. Disponible en: <https://www.jstor.org/stable/27733633?seq =1\#page_scan_tab_contents $>$. Acceso en: 6 nov. 2018.

ROBINSON, William. Una teoría sobre el capitalismo global. Bogotá: Ediciones desde abajo, 2007.

ROBINSON, William. América Latina y el capitalismo global. Una perspectiva crítica de la globalización. México: Siglo XXI Editores, 2015.

SOLO, Robert. The Neo-Marxist Theory of the State. Journal of Economic Issues, v. 12, n. 4, p. 829-842, 1978. Disponible en: <https://www.jstor.org/stable/4224747?seq= 1\#page_scan_tab_contents>. Acceso en: 6 nov. 2018.

SOTELO, Antonio; LAGNADO, Jacob. Sub-Imperialism revisited. Dependency theory in the thought of Ruy Mauro Marini. Boston: Brill, 2017.

THWAITES, Mabel; OUVIÑA, Hernan. La estatalidad latinoamericana revisitada. Reflexiones e hipótesis alrededor del problema del poder político y las transiciones. In: THWAITES, Mabel (Ed.). El Estado en América Latina: continuidades y rupturas. Santiago de Chile: CLACSO, 2012, p. 51-93.

TARCUS, Horacio (Comp.). Debates sobre el Estado capitalista. Estado y clase dominante. Buenos Aires: Imago Mundo, 1991.

TAPIA, Luis. La producción teórica para pensar América Latina. In: AGUILUZ, Maya; DE LOS RIOS, Norma (Coord.). René Zavaleta Mercado. Ensayos, testimonios y re-visiones. Buenos Aires: Flacso, 2006, p. 189-213.

TAPIA, Luis. La coyuntura de la autonomía relativa del Estado. La Paz: Muela del Diablo Editores Comuna - Clacso, 2009.

TORRES-RIVAS, Edelberto. Centroamérica: entre revoluciones y democracia. In: ROVIRA, Jorge (Comp.). Bogotá: Siglo del Hombre Editores, CLACSO, 2008.

VAN DER PIJL, Kees. Transnational Classes and International Relations. New York: Routledge, 1998.

WORLD BANK. World Development Report. World Economic Recession and Prospects for Recovery. Management in Development. World Development Indicators 1983. Disponible en: <http://documents.worldbank.org/curated/en/997591468322730301/pdf/111190REPLACEMEN T0WDR01983.pdfs. Acceso en: 6 nov. 2018. 
WORLD BANK. World Development Report. World Economic Recession and Prospects for Recovery. Management in Development. World Development Indicators 1985. Disponible en: <https://openknowledge.worldbank.org/handle/10986/5968>. Acceso en: 6 nov. 2018.

WORLD BANK. World Development Report. World Economic Recession and Prospects for Recovery. Management in Development. World Development Indicators 1986. Disponible en: <https://openknowledge.worldbank.org/handle/10986/5969>. Acceso en: 6 nov. 2018.

ZAVALETA, René. El Estado en América Latina. La Paz: Editorial Los Amigos del Libro, 1990.

ZELIK, Raúl. Paramilitarismo. Violencia y transformación social, política y económica en Colombia. Bogotá: Siglo del Hombre Editores - Fescol - Goethe Institut, 2015.

Texto recebido em 07 de novembro de 2018. Aprovado em 04 de fevereiro de 2019. 